\title{
Efecto de la endotelina-1 sobre las arterias tumorales de pacientes con neoplasia colorrectal
}

\author{
E. Ferrero Herrero, A. L. García Villalón', M. Labalde Martínez, G. Diéguez Castrillo \\ y M. Hidalgo Pascual
}

Servicio de Cirugía General y Digestivo “B”. Hospital Universitario 12 de Octubre. ${ }^{1}$ Departamento de Fisiología. Facultad de Medicina. Universidad Autónoma de Madrid

\begin{abstract}
RESUMEN
La endotelina-1 es un péptido vasoconstrictor producido por el endotelio vascular, cuyos niveles plasmáticos están aumentados en los pacientes con cáncer colorrectal y que puede participar en la regulación del flujo sanguíneo tumoral. Para estudiar si la respuesta a este péptido está alterada en las arterias tumorales, se obtuvieron, de 13 pacientes intervenidos quirúrgicamente por cáncer colorrectal, arterias mesentéricas irrigando el tumor y arterias mesentéricas de una región alejada del tumor, y asimismo se obtuvieron arterias mesentéricas de pacientes intervenidos por diverticulitis $(n=4)$ o enfermedad inflamatoria intestinal $(n=3)$. Las arterias mesentéricas se montaron en una preparación para el registro de la contracción isométrica en un baño de órganos, encontrándose que la endotelina-1 producía contracción en los tres tipos de arterias, pero la sensibilidad a este péptido fue mayor en las arterias irrigando el tumor que en las arterias alejadas del tumor o en las arterias de pacientes sin patología tumoral. Estos resultados indican que la endotelina-1 puede regular el flujo sanguíneo en los tumores colorrectales, produciendo una mayor vasoconstricción en las arterias que irrigan el tumor que en las arterias no tumorales.
\end{abstract}

Palabras clave: Endotelina-1. Cáncer colorrectal. Vasoconstricción. Endotelio.

\begin{abstract}
Endothelin-1 is an endothelium-derived vasoconstrictor peptide whose plasma levels are increased in patients with colorectal cancer, and which may be involved in tumor blood flow regulation. To study whether response to this peptide is altered in tumor arteries, mesenteric arteries supplying blood flow to colorectal tumors, and mesenteric arteries far from said tumors were obtained from 13 patients undergoing colectomy; mesenteric arteries were also obtained from patients with diverticulitis $(n=4)$ or inflammatory bowel disease $(n=3)$. Arteries were prepared for isometric tension recording in an organ bath, and in this preparation it was found that endothelin-1 induced contraction in all three types of arteries, but that sensitivity to this peptide was greater in arteries supplying blood flow to the tumor than in arteries far from the tumor or arteries from patients without cancer. These results suggest that endothelin-1 may regulate blood flow to colorectal tumors by inducing a greater contraction in tumor-supplying arteries than in non-tumor arteries.
\end{abstract}

Key words: Endothelin-1. Colorectal tumors. Vasoconstriction. Endothelium.

Ferrero Herrero E, García Villalón AL, Labalde Martínez M, Diéguez Castrillo G, Hidalgo Pascual M. Efecto de la endotelina-1 sobre las arterias tumorales de pacientes con neoplasia colorrectal. Rev Esp Enferm Dig 2008; 100: 327-331.

\section{INTRODUCCIÓN}

Los vasos sanguíneos que irrigan los tumores presentan alteraciones tanto morfológicas (1) como funcionales

Recibido: 26-12-07.

Aceptado: 26-02-08.

Correspondencia: E. Ferrero Herrero. Servicio de Cirugía General y Digestivo "B". Hospital Universitario 12 de Octubre. Avda. de Córdoba, s/n. 28041 Madrid.e-mail: eferrero.hdoc@salud.madrid.org
$(2,3)$ y estas características de los vasos tumorales pueden ser relevantes para favorecer el crecimiento y expansión del tumor, modificando el flujo sanguíneo al mismo. Uno de los factores que pueden estar implicados en la regulación del flujo sanguíneo en condiciones normales y patológicas es la endotelina-1, un péptido producido por las células endoteliales y que presenta un potente efecto vasoconstrictor (4). La endotelina-1 puede estar implicada en la regulación del flujo sanguíneo tumoral, puesto que sus niveles plasmáticos están aumentados en pacien- 
tes con cáncer colorrectal (5) y la respuesta vasconstrictora a este péptido está alterada en las arterias de tumores en ratas (6) o ratones (7).

Los vasos sanguíneos tumorales son un posible objetivo terapéutico en el tratamiento del cáncer, por lo que el conocimiento de la respuesta de estos vasos a los factores vasoactivos como la endotelina-1 puede tener interés. Actualmente no existe, que sepamos, ningún estudio de la respuesta a la endotelina-1 en los vasos tumorales humanos, puesto que los estudios existentes se han realizado en tumores experimentales de animales (8). Por tanto, el objetivo de este trabajo ha sido analizar la respuesta vasoconstrictora a la endotelina-1 de arterias procedentes de tumores colorrectales humanos, comparándola con la de arterias irrigando colon normal en los mismos pacientes, o con la de arterias procedentes de pacientes sin patología tumoral.

\section{MATERIAL Y MÉTODOS}

\section{Obtención de las arterias}

En este estudio se recogieron arterias mesentéricas (aproximadamente $1 \mathrm{~mm}$ de diámetro externo) de 13 pacientes intervenidos mediante colectomías por cáncer colorrectal, obteniendo arterias que irrigaban directamente el tumor y arterias alejadas del tumor que irrigaban colon normal. Así mismo, se obtuvieron arterias mesentéricas de pacientes en los que se practicaron colectomías por enfermedades no tumorales, como diverticulitis (4 pacientes) o enfermedad inflamatoria intestinal (3 pacientes). Las arterias se disecaban inmediatamente después de la extracción de la pieza y se introducían en solución salina isotónica fría para su traslado desde los quirófanos del Hospital Universitario 12 de Octubre al laboratorio del Departamento de Fisiología de la Facultad de Medicina de la Universidad Autónoma de Madrid.

Las arterias mesentéricas así obtenidas se limpiaban de tejidos adyacentes bajo un microscopio de disección (Fig. 1), y se cortaban en segmentos de $2 \mathrm{~mm}$ de longitud.

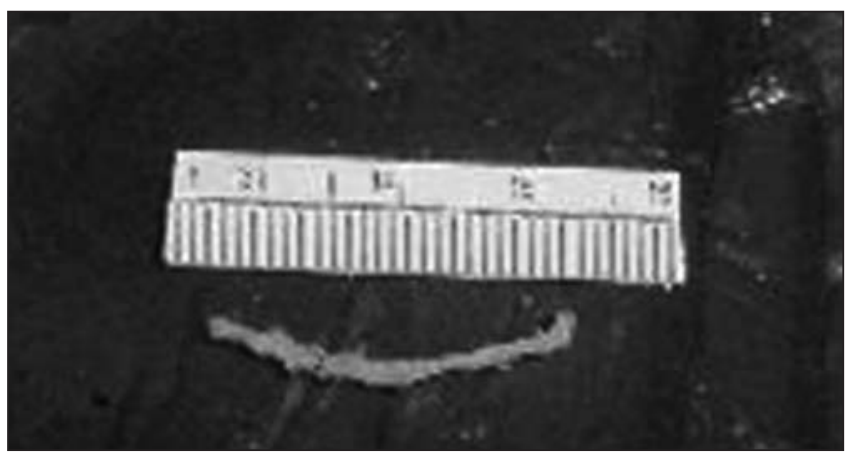

Fig. 1. Fotografía de una arteria mesentérica humana disecada de la pieza quirúrgica

\section{Registro de la tensión isométrica}

Para el registro de la tensión isométrica desarrollada por la pared de los segmentos arteriales, se introducían a través de la luz vascular dos alambres de tungsteno de $100 \mu \mathrm{m}$ de diámetro. Uno de los alambres quedaba unido a un soporte fijado a la pared del baño de órganos y el otro alambre, que era móvil, se conectaba a un transductor para el registro de la tensión isométrica (Universal Transducing Cell Statham microscale accesory UCL5). Un tornillo micrométrico, conectado al alambre móvil, permitía desplazar este alambre verticalmente en sentido perpendicular al eje mayor del segmento arterial y aplicar la tensión pasiva deseada a la pared arterial. El registro de la tensión se realizó en un ordenador mediante un sistema de adquisición de datos Maclab 8/e (AD Instruments) con el programa Chart V 3/4.

Cada segmento arterial, montado en el sistema explicado anteriormente, se colocaba en un baño de órganos que contenía $4 \mathrm{ml}$ de solución Krebs-Henseleit, cuya composición es la siguiente (mM): $\mathrm{NaCl} \mathrm{115;} \mathrm{KCl} 4,6$; $\mathrm{KH}_{2} \mathrm{PO}_{4} 1,2 ; \mathrm{SO}_{4} \mathrm{Mg} 1,2 ; \mathrm{CaCl}_{2} 2,5 ; \mathrm{NaHCO}_{3} 25 ;$ glucosa 11. Esta solución se equilibraba con una mezcla gaseosa $\left(95 \% \mathrm{O}_{2}\right.$ y $\left.5 \% \mathrm{CO}_{2}\right)$ para proporcionarle un $\mathrm{pH}$ 7,3-7,4, que se comprobaba con un $\mathrm{pH}$-metro de laboratorio, modelo Crison. Un circuito de agua caliente que rodeaba al baño permitió mantener la solución de Krebs-Henseleit junto con los segmentos vasculares a una temperatura de $37 \pm 0,5^{\circ} \mathrm{C}$ (Fig. 2).

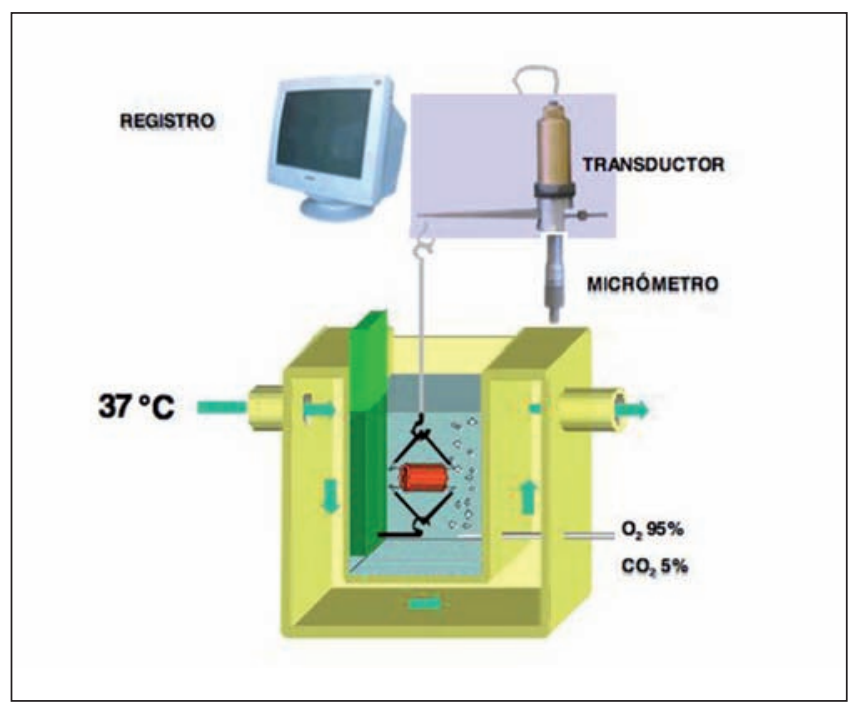

Fig. 2. Representación esquemática del montaje experimental utilizado para el registro de la tensión isométrica.

\section{Determinación de la tensión óptima}

Para determinar la tensión óptima de contracción se registró la respuesta a la serotonina $\left(10^{-5} \mathrm{M}\right)$ a distintas tensiones pasivas. Se utilizó la serotonina para esta determi- 
nación porque producía una marcada contracción de las arterias mesentéricas, que era reproducible cuando se estimulaba repetidamente el mismo segmento vascular. Tras montar los segmentos vasculares en el baño de órganos, se estiraba la pared vascular separando los dos alambres mediante el tornillo micrométrico, hasta que la tensión pasiva registrada mediante el transductor de tensión se estabilizaba en valores de 0,$15 ; 0,3 ; 0,6 ; 2 ; 4$ y $8 \mathrm{~g}$. A continuación se añadía serotonina al baño de órganos a una concentración de $10^{-5} \mathrm{M}$ y se registraba la contracción del músculo liso. Cuando esta contracción alcanzaba una meseta, se eliminaba la serotonina del baño renovando repetidamente la solución de Krebs-Henseleit y los segmentos se estiraban a la siguiente tensión pasiva. Puesto que la respuesta a la serotonina fue máxima a una tensión pasiva de 2-4 g, los experimentos posteriores se realizaron a una tensión de $2 \mathrm{~g}$.

\section{Respuesta a la endotelina-1}

Tras montar en el baño de órganos los segmentos vasculares de arterias mesentéricas, se ajustaba la tensión pasiva al valor previamente determinado de $2 \mathrm{~g}$ y se mantenían a esa tensión durante 2-3 horas hasta que se equilibraban. A continuación se estudiaba la respuesta a la endotelina-1 realizando curvas de concentración-respuesta acumulativas a esta sustancia. La endotelina- 1 se añadía al baño de órganos en concentraciones crecientes $\left(10^{-10}-10^{-7} \mathrm{M}\right)$ y después de añadir cada concentración se esperaba 10-15 min hasta que la contracción de la pared vascular alcanzaba una meseta, antes de añadir la siguiente concentración.

\section{Análisis de los datos}

La contracción a la serotonina o a la endotelina-1 se registraba como el aumento de la tensión por encima de la tensión basal al añadir dichas sustancias. En cada curva de concentración-respuesta a la endotelina-1 se calculó la $\mathrm{pD}_{2}$ como el logaritmo negativo de la concentración que producía una contracción igual al 50\% de la concentración máxima, obtenida por interpolación geométrica en la curva. La respuesta a la endotelina- 1 en los distintos grupos de arterias se comparó mediante análisis de la varianza, seguida de test de Bonferroni para determinar qué diferencias eran estadísticamente significativas. Se tomó como significativa una probabilidad menor de 0,05 . Los resultados se expresan como media \pm error estándar.

\section{RESULTADOS}

La serotonina produjo una contracción máxima de los segmentos vasculares de 1,46 $\pm 0,34 \mathrm{~g}$, obteniéndose una respuesta óptima cuando los segmentos vasculares estaban estirados a una tensión pasiva de 2-4 g y que era menor cuando los segmentos vasculares eran estirados a tensiones pasivas menores $(0,15 ; 0,3 ; 0,6 \mathrm{~g})$ o mayores $(8 \mathrm{~g})$ (Fig. 3$)$.

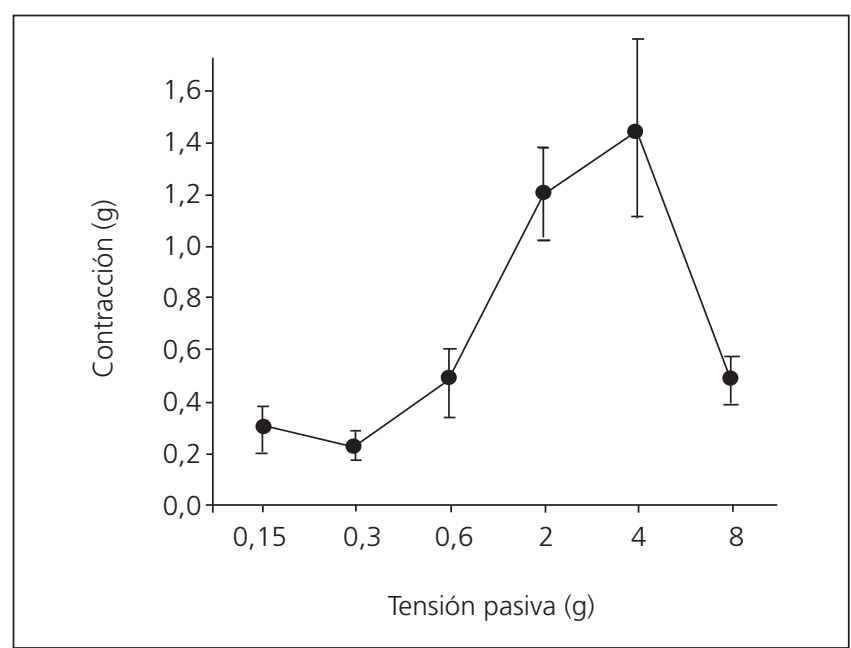

Fig. 3. Contracción a la serotonina $\left(10^{-5} \mathrm{M}\right)$ en arterias mesentéricas humanas equilibradas a distintas tensiones pasivas. Los valores representan la media \pm el error estándar obtenida en 16 segmentos.

La estimulación con endotelina-1 produjo contracción de las arterias mesentéricas dependiente de la concentración en todos los casos. La contracción máxima fue semejante en las arterias procedentes del tumor $(4,57 \pm$ $0,49 \mathrm{~g}$ ), en las arterias irrigando una región alejada del tumor en los mismos pacientes $(4,17 \pm 0,52 \mathrm{~g})$ y en las arterias de pacientes sin patología tumoral $(4,97 \pm 0,39 \mathrm{~g})$. Sin embargo, la sensibilidad $\left(\mathrm{pD}_{2}\right)$ fue mayor en las arterias irrigando el tumor $(8,21 \pm 0,13)$ que en las arterias de una región alejada en los mismos pacientes $(7,78 \pm 0,13$, $\mathrm{p}<0,05)$, o que en las arterias procedentes de pacientes sin patología tumoral $(7,80 \pm 0,08, \mathrm{p}<0,05)$ (Figs. 4 y 5$)$.

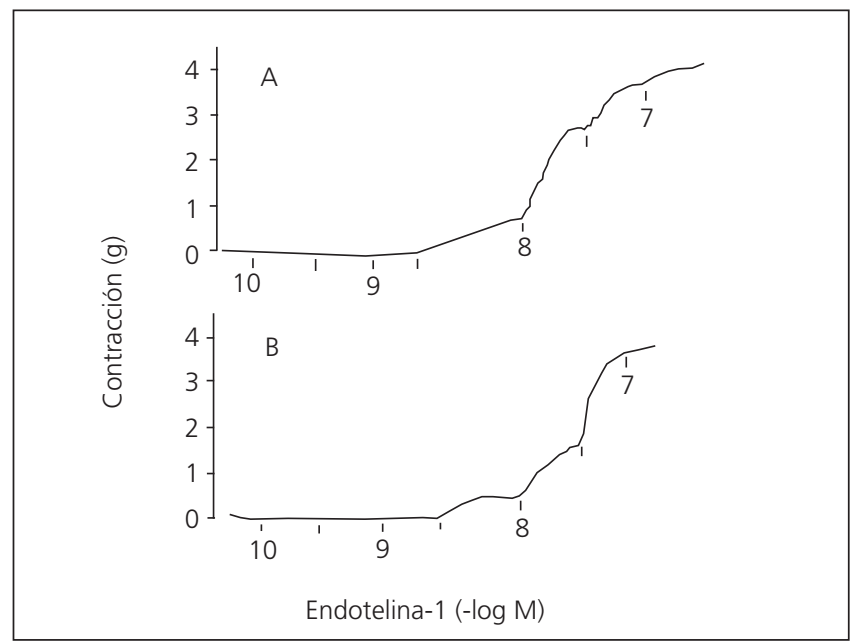

Fig. 4. Registros experimentales de la contracción a la endotelina-1 en una arteria procedente de un tumor colorrectal (A) y en una arteria procedente del mismo paciente alejada del tumor (B). 


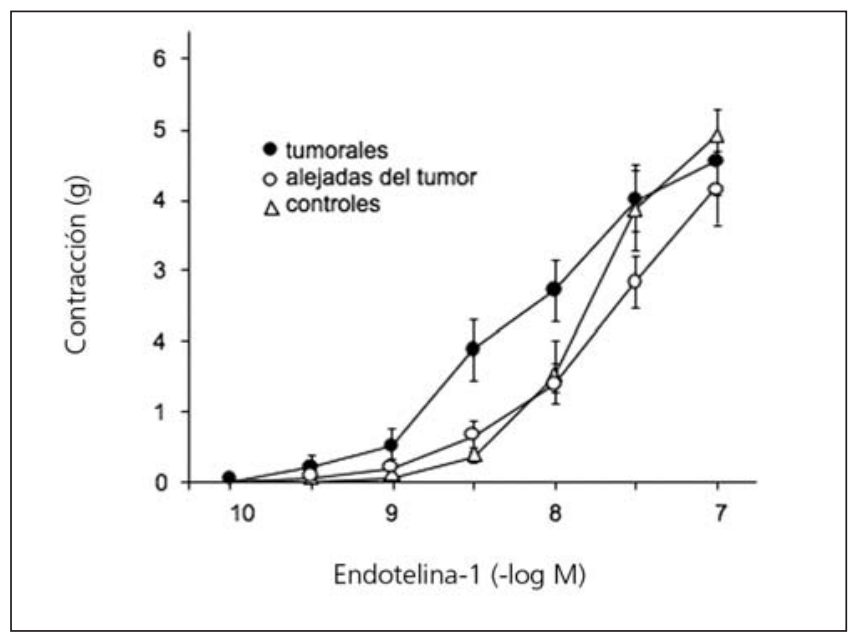

Fig. 5. Contracción a la endotelina-1 en arterias mesentéricas humanas obtenidas de tumores colorrectales (tumorales, 20 segmentos), de los mismos pacientes en una región alejada del tumor (16 segmentos), o procedentes de pacientes sin patología tumoral (control, 10 segmentos). Los valores representan la media \pm el error estándar.

\section{DISCUSIÓN}

Los resultados obtenidos en este estudio sugieren que la endotelina-1 produce una marcada contracción de las arterias irrigando tumores colorrectales, que puede ser mayor que en las arterias mesentéricas no tumorales.

Primeramente se determinó la tensión óptima de contracción en los segmentos vasculares, registrando la contracción a la serotonina, que produce activación del músculo liso vascular. La contracción a la serotonina fue máxima cuando los segmentos vasculares estaban estirados a una tensión pasiva de 2 ó 4 g y la respuesta disminuía cuando el estiramiento era menor o mayor. Esta dependencia de la tensión pasiva inicial para la respuesta contráctil es una característica del músculo, tanto liso como esquelético, y puede deberse a que el grado de solapamiento de los filamentos contráctiles varía con la longitud de las fibras musculares, siendo óptimo para un grado de estiramiento intermedio (9). La longitud que presentan los músculos in vivo es cercana a la óptima, porque en los estudios in vitro se utilizó esa longitud para reproducir en la medida de lo posible las condiciones fisiológicas. En este estudio, aunque la contracción a la serotonina fue ligeramente superior a 4 que a $2 \mathrm{~g}$, la diferencia no fue estadísticamente significativa y, puesto que una tensión pasiva alta durante un tiempo prolongado puede dañar la pared vascular, se seleccionó la tensión pasiva de $2 \mathrm{~g}$ para el resto de los experimentos.

La endotelina-1 produjo una contracción muy marcada de las arterias mesentéricas, tanto de las arterias que irrigaban el tumor como de las no tumorales. Esta contracción a la endotelina-1 fue mucho mayor que la producida por la serotonina, a pesar de que la serotonina es considerada un potente constrictor del músculo liso vascular. La respuesta encontrada en nuestro estudio es semejante a la descrita por Miyauchi y cols. (10) en arterias mesentéricas humanas y también a la encontrada en otras especies como ratas (11) o perros (12). El objetivo del presente estudio era analizar la respuesta a la endotelina-1 en las arterias tumorales y se encontró que la respuesta a este péptido fue mayor en las arterias irrigando tumores colorrectales que en las arterias irrigando colon normal en los mismos pacientes, o en las arterias de pacientes sin patología tumoral. Aunque la contracción máxima a concentraciones altas $\left(10^{-7} \mathrm{M}\right)$ de endotelina-1 fue semejante en los tres tipos de arterias, la sensibilidad $\left(\mathrm{pD}_{2}\right)$ fue mayor en las arterias tumorales que en los otros dos grupos. Esta diferencia puede tener relevancia fisiológica, puesto que la $\mathrm{pD}_{2}$ indica una mayor respuesta a las concentraciones bajas de endotelina-1 y son estas concentraciones bajas las que pueden darse en los tejidos en condiciones fisiológicas. Este incremento en la respuesta a la endotelina-1 encontrado en nuestro estudio podría deberse a factores locales liberados por las células tumorales y que afecten a los vasos sanguíneos cercanos, puesto que citoquinas como el factor de necrosis tumoral (TNF) o la interleuquina-1 beta aumentan la contracción en respuesta a la estimulación de los receptores a la endotelina-1 en arterias humanas $(13)$ o de rata $(14,15)$. Aunque las concentraciones plasmáticas de citoquinas pueden estar aumentadas en pacientes con diferentes tipos de tumor (16), las concentraciones locales de esta citoquina en el tejido tumoral pueden ser más relevantes que los niveles circulantes (17). En el presente estudio, la sensibilidad a la endotelina-1 estaba aumentada en las arterias irrigando los tumores colorrectales, pero no en las arterias de los mismos pacientes alejadas del tumor, puesto que la respuesta a la endotelina- 1 en estas últimas arterias fue semejante a la de arterias procedentes de pacientes sin patología tumoral. Esto sugiere que el cambio en la respuesta a la endotelina-1 puede deberse a las condiciones locales existentes en la proximidad del tumor y no a factores circulantes. Posteriores estudios serán necesarios para analizar si estas condiciones locales producen un incremento en los receptores vasculares a la endotelina-1.

En resumen, los resultados de este estudio sugieren que las arterias irrigando los tumores colorrectales son muy sensibles a la endotelina-1 y esto, unido a los niveles elevados de este péptido en los pacientes, producirá una vasoconstricción marcada en estas arterias. Se han propuesto estrategias terapéuticas dirigidas a aumentar el flujo sanguíneo tumoral, con el fin de facilitar la llegada de los fármacos antitumorales a las células tumorales, por lo que los antagonistas de los receptores para la endotelina-1 podrían ser útiles en este sentido.

\section{BIBLIOGRAFÍA}

1. Yaegashi H, Takahashi T. Encasement and other deformations of tumor-embedded host arteries due to loss of medial smooth muscles. Morphometric and three-dimensional reconstruction studies on some human carcinomas. Cancer 1990; 65: 1097-103. 
2. Suzuki M, Hori K, Saito S, Tanda S, Abe I, Sato H. Functional characteristics of tumor vessels: Selective increase in tumor blood flow. Sci Rep Res Inst Tohoku Univ 1989; 36: 37-45.

3. Nomura T, Ikezaki K, Natori Y, Fukui M. Altered response to histamine in brain tumor vessels: The selective increase of regional cerebral blood flow in transplanted rat brain tumor. J Neurosurg 1993; 79: 722-8.

4. Marasciulo FL, Montagnani M, Potenza MA. Endothelin: The ying and yang of vascular function. Curr Med Chem 2006; 13: 1655-65.

5. Arun C, London NJ, Hemingway DM. Prognostic significance of elevated endothelin-1 levels in patients with colorectal cancer. Int J Biol Markers 2004; 19: 32-7.

6. Bell KM, Prise VE, Chaplin DJ, Wordsworth S, Tozer GM. Vascular response of tumour and normal tissues to endothelin-1 following antagonism of $\mathrm{ET}_{\mathrm{A}}$ and $\mathrm{ET}_{\mathrm{B}}$ receptors in anesthetized rats. Int $\mathrm{J}$ Cancer 1997; 73: 283-9.

7. Soveaux P, Dessy C, Martinive P, Havaux X, Jordan BF, Gallez B, et al. Endothelin-1 is a critical mediator of myogenic tone in tumor arterioles: Implications for cancer treatment. J Cancer Res 2004; 64: 3209-14.

8. Noguera Aguilar JF, Gamundi Gamundi A. Experimental colonic carcinogenesis. Rev Esp Enferm Dig 2006; 98: 637-43.

9. Guyton AC, Hall JE. Contracción del músculo esquelético. En: Tratado de Fisiología Médica. 11ª ed. Madrid: Elsevier España; 2006. p. $72-91$.
10. Miyauchi T, Tomobe Y, Ishikawa T, Goto K, Sugishita Y. Vasoconstriction by endothelin-1 in resistance and conduit portions of isolated human mesenteric arteries. Eur J Pharmacol 1996; 303: 193-6.

11. Deng LY, Schiffrin EL. Calcium dependence of effects of endothelin on rat mesenteric microvessels. Can J Physiol Pharmacol 1991; 69: 798-804.

12. Tanoi C, Suzuki Y, Shibuya M, Sugita K, Masuzawa-Ito K, Asano M. Comparison of vasoconstrictor actions of endothelin-1 in cerebral, coronary, and mesenteric arteries of the dog. J Cardiovasc Pharmacol 1992; 19: 568-79.

13. White LR, Leseth KH, Moller S, Juul R, Adner M, Cappelen J, et al. Interleukin-1 beta potentiates endothelin ET(B) receptor-mediated contraction in cultured segments of human temporal artery. Regul Pept 1999; 31: 89-95.

14. Leshet KH, Adner M, Berg HK, White LR, Aaslly J, Edvinsson L. Cytokines increase endothelin $\mathrm{ET}_{\mathrm{B}}$ receptor contractile activity in rat cerebral artery. Neuroreport 1999; 10: 2355-9.

15. Udman E, Moller S, Adner M, Edvinsson L. Cytokines induce increased endothelin ET(B) receptor-mediated contraction. Eur J Pharmacol 1999; 376: 223-32.

16. Caro-Patón Gómez A. Interleukins and colon cancer. Rev Esp Enferm Dig 2005; 91: 619-28.

17. Yan L, Anderson GM, DeWitte M, Nakada MT. Therapeutic potential of cytokine and chemokine antagonists in cancer therapy. Eur J Cancer 2006; 42: 793-802. 